\title{
ATIVIDADE OVICIDA DE DOIS FÁRMACOS EM CAPRINOS NATURALMENTE PARASITADOS POR NEMATÓDEOS GASTRINTESTINAIS, RS, BRASIL
}

\author{
OVICIDAL ACTIVITY OF TWO MEDICAMENTS AGAINST GOAT GASTROINTESTINAL \\ NEMATODE IN RS, BRAZIL
}

\author{
Mary Jane Tweedie de Mattos ${ }^{1}$ Verônica Schmidt ${ }^{2}$ Cristine Dossin Bastos ${ }^{3}$
}

\section{- NOTA -}

RESUMO

\begin{abstract}
A eficácia comparativa entre levamisole em duas doses diferentes e closantel foi avaliada sobre ovos de nematódeos gastrintestinais de caprinos naturalmente parasitados. Observou-se que a redução de ovos de nematódeos gastrintestinais foi de 93,23\%, 91,25\% e 70,42\% nos grupos medicados com levamisole $8 \mathrm{mg} / \mathrm{kg}$, levamisole $11 \mathrm{mg} / \mathrm{kg} e$ closantel $10 \mathrm{mg} / \mathrm{kg}$, respectivamente. O teste de desenvolvimento embrionário revelou que levamisole, nas duas doses, foi eficaz sobre Haemonchus spp., Ostertagia spp., Cooperia spp. e Oesophagostomum spp. O closantel não foi eficaz para Cooperia spp e Oesophagostomum spp.
\end{abstract}

Palavras-chave: caprinos, anti-helminticos, nematódeos gastrintestinais.

\section{SUMMARY}

The efficacy of the anthelmintics levamisole in two different doses $(8 \mathrm{mg} / \mathrm{kg}$ and $11 \mathrm{mg} / \mathrm{kg})$ and closantel $(10 \mathrm{mg} / \mathrm{kg})$ were compared against gastrointestinal nematodes in naturally infected goats. The reduction on the faecal egg count was $93.23 \%$ in the group treated with levamisole at the dose of $8 \mathrm{mg} / \mathrm{kg}$, $91.25 \%$ in the group treated with the dose of $11 \mathrm{mg} / \mathrm{kg}$ and $70.42 \%$ in the group treated with closantel. The anthelmintic levamisole was effective against Haemonchus spp., Ostertagia spp., Cooperia spp and Oesophagostomum spp. However closantel wasn't effective against Cooperia spp and Oesophagostomum spp.
Key words: goat, anthelmintic, gastrointestinal nematode.

A caprinocultura vem se expandindo nos últimos anos e um dos problemas enfrentados pelos criadores é o controle das doenças parasitárias, em particular as helmintoses gastrintestinais Como forma de controle das helmintoses, os criadores utilizam anti-helminticos nas mesmas doses daqueles preconizados para ovinos. Na literatura, estão registrados vários trabalhos de pesquisa relativos a eficácia dos benzimidazóis sobre nematódeos gastrintestinais de ovinos e caprinos (WALLER, 1986; COLES \& ROUSCH, 1992; CABARET, 1995). Em relação especificamente aos caprinos, estão descritos os experimentos de GUHA \& BANERJEE (1987), COLES et al.(1989), MWAMACHI et al. (1995), MAINGI et al.(1996) e HONG et al. (1996) que testaram levamisole e os benzimidazóis. A eficácia do closantel foi verificada por MWAMACHI $\boldsymbol{e t}$ al. (1995), em ovinos no Kenya, por UPPAL et al. (1993) em caprinos, na India e por DORNY et al. (1994) em caprinos da Malasia. No Brasil, vários pesquisadores verificaram a ação anti-helmíntica do levamisole, albendazole e parbendazole em caprinos naturalmente parasitados por nematódeos gastrintes-

\footnotetext{
${ }^{1}$ Médico Veterinário, Professor Adjunto, Faculdade de Veterinária, Universidade Federal do Rio Grande do Sul (UFRGS), Av.Bento Gonçalves, 9090, 91540-000, Porto Alegre - RS. E-mail: maryvet@ vortex.ufrgs.br

${ }^{2}$ Médico Veterinário, Professor Assistente, Faculdade de Veterinária, UFRGS.

${ }^{3}$ Médico Veterinário, Mestrando do Programa de Pós-graduação em Ciências Veterinárias da Faculdade de Veterinária, UFRGS. 
tinais, como Vieira (1986) apud VIEIRA e CAVALCANTE (1998) no Estado do Ceará, CHARLES $\boldsymbol{e t}$ al.(1989), em Petrolina e SANTOS $\boldsymbol{e t}$ al. (1993) também em Pernambuco. Em relação ao closantel em caprinos, não há relatos no Brasil. $\mathrm{O}$ experimento foi desenvolvido no município de Gravataí-RS com o objetivo de verificar a eficácia de closantel e levamisole em caprinos adultos, naturalmente parasitados por nematódeos gastrintestinais.

A propriedade selecionada localiza-se no município de Gravataí (Grande Porto Alegre), sendo o plantel composto por 60 caprinos da raça anglonubiana. O regime de criação é do tipo semi-extensivo, onde os animais são recolhidos no galpão só à noite. Os animais, num total de 40 caprinos adultos, com peso médio de $40 \mathrm{~kg}$, foram selecionados baseados no exame parasitológico de fezes e separados em 4 grupos. No grupo A, os caprinos receberam closantel $10 \mathrm{mg} / \mathrm{kg}$ via oral; no grupo $\mathrm{B}$, os caprinos receberam levamisole $8 \mathrm{mg} / \mathrm{kg}$ via subcutânea; no grupo C, os caprinos receberam levamisole $11 \mathrm{mg} / \mathrm{kg}$ via subcutânea; e, no Grupo D (controle), os animais receberam placebo. Amostras fecais dos animais foram colhidas diretamente do reto, no dia da medicação (dia zero) e sete dias após, sendo processadas pelo método de Gordon \& Withlock modificado com a câmara de McMaster e Robert's O'Sullivan apud UENO \& GONÇALVES, 1998. Além disso, foram colhidas amostras fecais de todos os caprinos a cada duas horas. A eficácia dos anti-helmínticos foi realizada baseada na redução do opg (ovos por grama de fezes) comparando os dias zero e sete.

Os resultados evidenciaram uma redução do opg (ovos de nematódeos gastrintestinais por grama de fezes) de 70,42\%, 93,30\% e 91,25\% nos grupos $\mathrm{A}, \mathrm{B}$ e $\mathrm{C}$, respectivamente (Tabela 1). Não houve diferença significativa entre as doses de levamisole. A coprocultura do dia zero revelou que a prevalência foi maior de larvas do gênero Haemonchus spp., nos grupos A, B e C e de Ostertagia spp. no grupo D (Tabela 2). No sétimo dia após a medicação, não houve desenvolvimento de larvas nos grupos B e C, embora tenham sido observados ovos de Strongyloidea (média de opg foi de 90 e 70, respectivamente) estes não eram viáveis. No grupo A, foram observadas larvas de Cooperia spp. (85\%), e Oesophagostomum spp. (15\%) e, no grupo D, foram observadas larvas de Haemonchus (42\%), Cooperia (28\%) Ostertagia spp. (25\%) e Oesophagostomum spp. (5\%). O Closantel (grupo A) funcionou bem sobre Haemonchus spp. e Ostertagia spp., pois não houve desenvolvimento de larvas desses gêneros no sétimo dia após a medicação. Em relação a Cooperia spp. e Oesophagostomum spp, ele não foi eficaz, pois
Tabela 1 - Eficácia do Closantel e Levamisole em caprinos naturalmente infectados por nematódeos gastrintestinais.

\begin{tabular}{llll}
\hline \multicolumn{4}{c}{ Média ovos de Strongyloidea } \\
Grupo & dia zero & setimo dia & Redução(\%) \\
\hline & 1420 & 420 & 70,42 \\
A & $(500-3900)$ & $(100-1800)$ & \\
& 1330 & 90 & 93,23 \\
B & $(500-3100)$ & $(100-500)$ & \\
& 800 & 70 & 91,25 \\
C & $(500-2400)$ & $(100-200)$ & \\
& 1625 & 1718 \\
D & $(500-4600)$ & $(500-4700)$
\end{tabular}

A-Closantel 10mg/kg, B-Levamisole $8 \mathrm{mg} / \mathrm{kg}, \quad$ C-Levamisole $11 \mathrm{mg} / \mathrm{kg}$, D-Controle.

houve desenvolvimento de larvas após a medicação. Com relação à presença de ovos nas fezes dos animais, verificou-se que após 6 horas do tratamento realizado, os animais ainda expeliam ovos de gastrintestinais viáveis, o que pode ser comprovado pelas coproculturas realizadas (Tabela 2). Os resultados das coproculturas revelaram que os gêneros existentes nos diferentes grupos foram os mesmos, variando apenas suas prevalências. $\mathrm{O}$ Haemonchus foi o gênero que já na coleta das 2 horas se manteve como o mais prevalente em todos

Tabela 2 - Gêneros de nematódeos gastrintestinais identificados no teste de desenvolvimento embrionário zero, duas, quatro, seis e 168 horas (\%).

\begin{tabular}{|c|c|c|c|c|c|c|}
\hline GRUPO & GENERO & $\mathrm{Oh}$ & $2 \mathrm{~h}$ & $4 \mathrm{~h}$ & $6 \mathrm{~h}$ & $168 \mathrm{~h}$ \\
\hline \multirow[t]{4}{*}{ A } & Haemonchus & 40 & 35 & 40 & 40 & 0 \\
\hline & Ostertagia & 30 & 25 & 30 & 20 & 0 \\
\hline & Cooperia & 20 & 30 & 20 & 30 & 85 \\
\hline & Oesophagostomum & 10 & 10 & 10 & 10 & 15 \\
\hline \multirow[t]{4}{*}{ B } & Haemonchus & 50 & 65 & 60 & 50 & 0 \\
\hline & Ostertagia & 20 & 5 & 10 & 30 & 0 \\
\hline & Cooperia & 20 & 0 & 0 & 0 & 0 \\
\hline & Oesophagostomum & 10 & 30 & 30 & 20 & 0 \\
\hline \multirow[t]{4}{*}{$\mathrm{C}$} & Haemonchus & 48 & 40 & 49 & 100 & 0 \\
\hline & Ostertagia & 32 & 40 & 20 & 0 & 0 \\
\hline & Cooperia & 10 & 10 & 10 & 0 & 0 \\
\hline & Oesophagostomum & 10 & 10 & 10 & 0 & 0 \\
\hline \multirow[t]{4}{*}{$\mathrm{D}$} & Haemonchus & 30 & 29 & 40 & 35 & 42 \\
\hline & Ostertagia & 40 & 40 & 30 & 32 & 25 \\
\hline & Cooperia & 20 & 21 & 23 & 19 & 28 \\
\hline & Oesophagostomum & 10 & 10 & 7 & 14 & 5 \\
\hline
\end{tabular}

Ciência Rural, v. 30, n. 5, 2000. 
os grupos, até a coleta das 6 horas. Os dados da presente pesquisa se aproximam das observações realizadas por SANTOS et al. (1993) que verificaram uma eficácia de $93,6 \%$ sobre Haemonchus spp., embora tenham utilizado uma dose de LBZ maior. O mesmo autor, quando utilizou cloridrato de levamisole na dose de $7,5 \mathrm{mg} / \mathrm{kg}$ verificou que ele não tinha efeito sobre Oesophagostomum spp. atribuindo a isto o uso de uma cepa resistente ao levamisole. Em relação ao closantel, UPPAL $\boldsymbol{e t}$ al. (1993) e DORNY $\boldsymbol{e t}$ al.(1994) observaram que esse princípio ativo na dose de $10 \mathrm{mg} / \mathrm{kg}$ apresentou uma eficácia de $100 \%$ sobre Haemonchus spp. que coincide com o presente estudo (Tabela 1). Os resultados do presente estudo permitem concluir que a dose de levamisole a ser usada no caso de infecções mistas pelos gêneros Haemonchus, Ostertagia, Cooperia e Oesophagostomum é de $8 \mathrm{mg} / \mathrm{kg}$, não havendo diferença significativa com a dose de $11 \mathrm{mg} / \mathrm{kg}$. Além disso, o closantel a $10 \mathrm{mg} / \mathrm{kg}$ foi eficaz sobre Haemonchus spp. e Ostertagia spp. Finalmente, para que os anti-helmínticos tenham efeito ovicida é necessário que os caprinos permaneçam, pelo menos, 6 horas presos antes de serem soltos após a medicação.

\section{REFERÊNCIAS BIBLIOGRÁFICAS}

CABARET, J., BAUDET, H.M., DEVOS, J., et al. Studies on multispecific resistance of gastrointestinal nematodes to benzimidazoles on dairy-goat farms. Vet Parasitol, v.60 p.331-337,1995.

COLES, G.C., GIORDANO, D.J., TRITSCHLER, J.P. Efficacy of levamisole against immature and mature nematodes in with induced infections. Am J Vet Res v.50, n.7, p.1074$1075,1989$.

COLES, G.C., ROUSH, R.T. Slowing the spread of anthelminthic resistant nematodes of sheep and goats in the United Kingdom. Vet Rec. v.6, p.505-510, 1992

CHARLES, T.P., POMPEU, J., MIRANDA, D.B. Efficacy of three broad-spectrum anthelmintics against gastrointestinal nematode infections of goats. Vet Parasitol, v.34, p.71-75, 1989.

DORNY, P., VERCRUYSSE, J., JALILA, A., et al. Control of haemonchosis in Malaysian goats with closantel. Vet Parasitol, v.53, n.3-4, p. 233-241,1994.

GUHA, C., BANERJEE, A.K. Efficacy of four anthelmintics in the treatment gastrointestinal nematodes of goats a comparative assessment. Indian Veterinary Journal,. v.64, p.335-337, 1987.

HONG, C., HUNT, K.R., COLES, G.C. Occurrence of anthelmintic resistant nematodes on sheep farms in England and goat farms in England and Wales. Veterinary Record, v. 27 p.83-85,1996.

MAINGI, N., BJORN, H., THOMSBORG, S.M., et al. A questionnaire survey of nematode parasite control practices on goat farms in Denmark. Veterinary Parasitology, v.66 p.25-37,1996 a.

MAINGI, N., BJORN, H., THOMSBORG, S.M., et al. A survey of anthelmintic resistance in nematode parasites of goats in Denmark. Veterinary Parasitology, v 66 p.53-66, 1996 b .

MWAMACHI, D.M., AUDHO, J.O., THORPE, W., $\boldsymbol{e}$ t al. Evidence for multiple anthelmintic resistance in sheep and goats reared under the same management in coastal Kenya. Veterinary Parasitology, v.60 p.303-313,1995.

SANTOS, N.V.M., CHARLES, T.P., MEDEIROS, E.M.A.M. Eficácia do cloridrato de levamisole em infestações por nematódeos gastrintestinais em caprinos. Arquivo Brasileiro Medicina Veterinaria e Zootecnia. v.45 p.487-495,1993.

UENO, H., GONÇALVES, P.C. Manual para o Diagnóstico de Helmintoses de ruminantes. 4. ed. Tóquio : Japan International Cooperation Agency, 1998. 143p.

UPPAL, R.P., YADAV, C.L.E., BHUSHAN, C. Efficacy of closantel against fenbendazole and levamisole resistant Haemonchus contortus in small ruminants. Tropical Animal Health Production, v.25 n.1 p.30-32, 1993.

VIEIRA, L. DA S., GONÇALVES, P.C., COSTA, C.A.E., et al. Redução e esterilização de ovos de nematódeos gastriontestinais em caprinos medicados com anti-helminticos benzimidazóis. Pesquisa Agropecuária Brasileira, v.24 n.10 p.1255$1265,1989$.

VIEIRA, L., CALVACANTE, A.C.R. Resistência antihelmintica em nematódeos gastrintestinais de caprinos. Revista Brasileira de Medicina Veterinária, v.20.n.3 p.112$117,1998$.

WALLER, P.J. Anthelmintic resistance in nematode parasites of sheep. Agricultural Zoology Research, v.1 p.333-373, 1986.

Ciência Rural, v. 30, n. 5, 2000. 